\title{
Short-arc analysis of intersatellite tracking data in a gravity mapping mission
}

\author{
David D. Rowlands ${ }^{1}$, Richard D. Ray ${ }^{1}$, Douglas S. Chinn ${ }^{2}$, Frank G. Lemoine ${ }^{1}$ \\ ${ }^{1}$ Space Geodesy Branch, NASA Goddard Space Flight Center, Greenbelt MD 20771, USA \\ ${ }^{2}$ Raytheon ITSS, Code 926, NASA/GSFC, Greenbelt, MD 20771, USA
}

Corresponding author:

David D. Rowlands

NASA/GSFC, Code 926

Greenbelt, MD 20771

USA

tel: (301) 614-6110

fax: (301) 614-6099

email: drowland@helmert.gsfc.nasa.gov

June 21, 2001 
Abstract. A technique for the analysis of low-low intersatellite range-rate data in a gravity mapping mission is explored. The technique is based on standard tracking data analysis for orbit determination but uses a spherical coordinate representation of the 12 epoch state parameters describing the baseline between the two satellites. This representation of the state parameters is exploited to allow the intersatellite range-rate analysis to benefit from information provided by other tracking data types without large simultaneous multiple data type solutions. The technique appears especially valuable for estimating gravity from short arcs (e.g. less than 15 minutes) of data. Gravity recovery simulations which use short arcs are compared with those using arcs a day in length. For a high-inclination orbit, the short-arc analysis recovers low-order gravity coefficients remarkably well, although higher order terms, especially sectorial terms, are less accurate. Simulations suggest that either long or short arcs of GRACE data are likely to improve parts of the geopotential spectrum by orders of magnitude.

Key words: Geopotential determination — Satellite geodesy — Satellite-to-satellite tracking GRACE.

\section{Introduction}

For more than three decades the geodetic community has realized that satellite-to-satellite tracking (hereinafter SST) provides extremely strong observational constraints for determining the geopotential (e.g. Wolff, 1969; Vonbun, 1972). High-low satellite configurations have proven,valuable in the past (e.g., Kahn et al., 1982), and they continue to do so today (Lemoine et al., 1998b; Schwintzer et al., 2000). Low-low satellite configurations are expected to yield orders of magnitude improvements in geopotential definition (National Research Council, 1997), and such a system may finally come to fruition in the near future with the Gravity Recovery and Climate Experiment (GRACE) mission 
(Tapley and Reigber, 2000).

Methods for deducing the geopotential from low-low SST data have been developed by many groups over the past two decades (e.g., Douglas et al., 1980; Kaula, 1983; Wagner, 1983, 1987; Colombo, 1984; Jekeli and Upadhyay, 1990). Many of these methods were based on semi-analytic theories that made various simplifying assumptions to overcome computational limitations. For example, both Kaula's and Colombo's methods assumed perfectly polar orbits-a "tail-biting orbit" was Colombo's colorful phrase-which allow, among other advantages, fast Fourier techniques. Computational limitations are still an important consideration today, but not nearly so much as when these earlier works were written.

This paper explores a direct approach to estimating gravity from SST data, relying more heavily on numerical integration methods than on analytic or semi-analytic methods. We present extensive simulations with the following SST scenario: near-polar low-low satellites, separated by a couple hundred kilometers, each satellite tracked continuously by GPS and each deployed with accelerometers to correct for non-conservative forces. The fundamental measurement is the satellite-to-satellite range rate, measured with a precision of order $10^{-6} \mathrm{~m} \mathrm{~s}^{-1}$.

In addition to establishing a viable technique for the handling SST data, this paper addresses two key questions for any practical data analysis:

1. To what extent is the SST gravity inversion insensitive to ephemeris accuracy? Specifically, is it sufficiently insensitive that the orbit determination and the gravity inversion can be performed in separate, independent steps?

2. To what extent is the SST gravity inversion dependent on arc length? In particular, if the accelerometers are incapable of removing all non-conservative forces, including thrusting events, the satellite data might necessarily be broken into very short arcs. How will the gravity estimation be affected? 
Concerning (1), our simulations described below show that, indeed, the SST data can be handled (within limitations) independently of the GPS data. We validate a two-step method. The first step concentrates only on achieving orbit accuracy (no gravity estimation). The second step uses only the SST data to refine certain components of the orbit while estimating gravity coefficients. A great advantage of this is that the orbit determination task in an SST mission can concentrate on ephemeris accuracy, using techniques such as empirical accelerations that are normally prohibited in standard satellite gravity estimation. A second practical advantage is, of course, that the gravity inversion task can be performed without the considerable simultaneous data processing chores associated with GPS orbit determination.

The next section compares the role of arc length in conventional orbit determination based gravity estimation with the role it may play in a GRACE-like SST mission. Section 3 describes a transformation that we apply to the standard orbit parameters to enable our decoupled analysis of the SST data. Section 4 gives the rationale behind our data simulation procedures as well as a detailed description of those procedures. Sections 5 and 6 present results of parameter estimations using simulated data. Section 5 concentrates on the estimation strategy for orbit parameters, while Section 6 presents the results of gravity field recoveries with orbit parameters adjusting simultaneously.

\section{Arc Length in Gravity Estimation}

When satellite tracking data are analyzed for geophysical parameter recovery, the analysis is often part of a simultaneous solution for orbit parameters. In such settings it is usually necessary to group the tracking data into "arcs" of data which span multiple revolutions of the satellite. This is because most tracking data types do not provide enough geometric strength to provide a unique solution for the orbit until a substantial portion of the trajectory is examined. Furthermore, force model parameters like gravity coefficients build up sensitivity with arc length, because gravity signal is usually detected through its effect on the trajectory of a satellite. If two trajectories for a satellite are computed, 
each starting from the same initial conditions but using different gravity models, it usually takes some period of time from the initial epoch before differences in the trajectories are large enough that differences in the gravity signal can be inferred. The trajectories are used to compute "theoretical" values of the tracking data observations to which the actual observations are compared. The difference in the trajectories due to gravity needs to affect the computation of the theoretical tracking data values above the level of the precision of the actual tracking data. Furthermore, the differences in trajectories computed with different gravity models are often diminished because the initial state estimation process at some level accommodates gravity errors. Gravity is really an "indirect effect" in conventional tracking data analysis. Arc length is required for the indirect effect to make its presence felt.

In general, long arcs are desirable. On the other hand, as the arc length grows, so does the effect of unmodeled forces. Therefore, the length of an arc in an orbit solution cannot be extended indefinitely without degrading the solution. Choice of arc length is a key decision in the analysis of tracking data. It depends on the geometric strength of the tracking data, the magnitude of unmodeled forces, and the sensitivity of the data to the geophysical parameters of interest. Gravity models like EGM96 (Lemoine et al., 1998a) have typically used tracking data analyzed in arcs of data from 1 to 30 days. With very few exceptions the tracking data used in EGM96 would not support the extraction of gravity signal from an arc of data significantly shorter than a day.

In a gravity mapping mission where there is very precise tracking of the change in range between two satellites in the same orbit plane, many of the conditions that have always been a factor in deciding arc length for conventional tracking data will be much different. The most obvious difference is the high precision of the data. Small trajectory changes are detectable with more precise data. Furthermore, the intersatellite observation is, in some sense, a direct measurement of the difference in forces which the satellites experience at each instant, and hence almost a direct measurement of gravity (Colombo, 
1984). The extraction of gravity signal from such a measurement is unlikely to require the type of trajectory analysis that conventional tracking data requires. A precise range change measurement between two satellites in the same orbit plane immediately senses the direct effect of gravity as well as the more latent indirect effect (effect on trajectory) that conventional tracking data relies upon exclusively.

The orbit determination aspects of a gravity mapping mission places different requirements on arc length as well. Precise range change measurements between two satellites do not provide sufficient information to determine independently the orbits of the two satellites. As will be demonstrated below, these measurements are extremely sensitive to some components of the orbits of the two satellites and almost completely insensitive to others (relative to how well they can be determined from other tracking data types). If the initial epoch state vectors of the two satellites are determined with other tracking data types, it should be possible to use the range change measurements by themselves to refine only certain components of the orbits. This has the potential to allow shorter arcs. Orbit refinement does not place the same constraints on arc length as orbit determination.

Even if the use of short arcs in a gravity mission is possible, the question remains if their use is desirable. As mentioned above, the effect of unmodeled forces places an upper limit on the length of arcs. In a mission which relies on high precision tracking, the tolerance for unmodeled forces is especially low. Accelerometry can reduce the level of unmodeled forces, but it remains to be seen how well accelerometry will mix with very precise tracking data. For example, small thrusting events may be inadequately sampled by accelerometers or there may be small data gaps or noise. These situations are most straightforwardly handled by analyzing short arcs that avoid periods of anomalous accelerations. Furthermore, it is possible that for some applications, short arcs are desirable. For example, short arcs may facilitate the independent analysis of regional data in the form of gravity anomaly blocks or some other local gravity parameterization. It is beyond the scope of this investigation to answer many of the 
questions surrounding the desirability of short arc analysis. We intend to demonstrate the possibility of short arc (under 15 minute in length) analysis. We also explore the tradeoff between using short arcs and arcs of more conventional length (1 day) assuming that the longer arcs are possible within the constraints presented by a real gravity mapping mission.

\section{Spherical Coordinates for Initial Epoch State Parameters}

Orbit solutions often solve for the six cartesian components of a satellite's state vector at an initial epoch. After each iteration of an orbit solution, the updated initial cartesian state vector is used to compute the trajectory at later epochs, which is accomplished by numerical integration of the cartesian components of the state vector. Even though the initial epoch state vector is required in cartesian form, it is sometimes useful to solve for an alternative representation of the initial state vector-for example, osculating Kepler elements. Often it is easier to apply useful constraint equations that are applicable to a particular data type when a non-cartesian representation is used. After the alternative form of the state vector is updated, it is transformed to cartesian coordinates and the numerical integration proceeds. It is straightforward to convert an orbit solution from a cartesian state vector solution to some other representation. All that is required is the six by six matrix of the partial derivatives of the six initial cartesian state vector parameters with respect to the six alternative parameters.

In the case of the two satellites of a gravity SST mission, the twelve parameters describing the two initial cartesian state vectors can be converted to the cartesian state vector of the baseline (the difference vector) between the two satellites and the cartesian state vector of the baseline midpoint (the average of the two satellite state vectors). These two vectors can be further transformed. In the case of the midpoint state vector, it is useful to convert the three position components to spherical coordinates: the latitude of the midpoint, the longitude of the midpoint and the radius of the midpoint. For the baseline vector, it is useful to imagine a local cartesian coordinate system centered at the midpoint of the baseline. The $X Y$ plane of the system is perpendicular to the position vector of the system 
midpoint. The $X$ axis is the local East vector of the local coordinate system. It is further useful to describe both the position and velocity components of the baseline state vector in spherical coordinates which are based in this local coordinate system. In each case, the vector is converted to magnitude, pitch (angle the vector makes with $X Y$ plane) and yaw (angle that the projection onto the $X Y$ plane makes with the $X$ axis). The twelve new epoch state parameters are:

$R_{m} \quad$ Distance of baseline midpoint from Earth center of mass

$\Phi_{m} \quad$ Geocentric latitude of baseline midpoint

$\Lambda_{m} \quad$ Longitude of baseline midpoint

$\dot{X}_{m} \quad$ Inertial $X$ component of baseline midpoint velocity vector

$\dot{Y}_{m} \quad$ Inertial $Y$ component of baseline midpoint velocity vector

$\dot{Z}_{m} \quad$ Inertial $Z$ component of baseline midpoint velocity vector

$R_{b p} \quad$ Baseline length

$P_{b p} \quad$ Baseline position pitch

$Y_{b p} \quad$ Baseline position yaw

$R_{b v} \quad$ Baseline velocity vector magnitude

$P_{b v} \quad$ Baseline velocity vector pitch

$Y_{b v} \quad$ Baseline velocity vector yaw

We have implemented the ability to solve for the above twelve parameters in our orbit determination and geodetic parameter estimation software, GEODYN (Pavlis et al., 2001). This required only a new subroutine which transforms back and forth between a pair of cartesian state vectors and the above twelve parameters and which also computes the twelve by twelve matrix of the partial derivatives of the twelve initial cartesian state parameters with respect to the twelve spherical coordinate parameters.

In the next sections we demonstrate that the above twelve parameters are promising for use in the 
analysis of the intersatellite range change measurements when they are decoupled from other tracking data types that might be available. One reasonably expects that the intersatellite range change measurement is more sensitive to the baseline parameters than to the midpoint parameters. One also expects that the intersatellite range change measurement is fairly insensitive to the baseline yaw parameters. If the range change measurements are to be analyzed independently from other tracking data types, then it is important to study the sensitivity of the range change measurement to each of the above twelve parameters. The sensitivity of the measurement to each of the twelve parameters should be compared with how well each parameter is likely to be determined independently from other tracking data types available in the mission. That subject is explored in Section 5.

\section{Data Simulation and Assumptions for Data Reduction}

All of the studies in this paper are based on using simulated one-way intersatellite range-rate data with a counting interval of 1 second. The data were generated using the orbits of two co-orbiting satellites with characteristics shown in Table 1. The key points are that the satellites are at an altitude very close to 500 kilometers, have a very low eccentricity, are very nearly polar and are separated by about 200 kilometers. The second satellite's initial elements are an exact copy of the first satellite's initial elements 30 seconds later. The satellite orbits were generated with the EGM96 gravity field using all terms up through degree 120 . Drag and solar radiation were not modeled in our simulations; it is assumed that accelerometry will sufficiently account for these forces. Accelerometry should also compensate for small thrusting events, but it is possible that thrusting can cause occasional problems, so our simulations do include thrusting. Each satellite was given a thrust every 30 minutes (a $\Delta V$ of about $0.5 \mathrm{~mm} \mathrm{~s}^{-1}$ ) but in a staggered manner so that the satellite-satellite system received a $\Delta V$ every 15 minutes. (This, in fact, is roughly comparable to the thrusting frequency currently occurring on the CHAMP satellite [Schwintzer, 2000].) Although it would be hoped that an accelerometer would accurately model thrusts, we wish to determine if problematic thrusts can be removed from 
the analysis process altogether (assuming they occur no more frequently than every 15 minutes). We therefore investigate whether gravity signal can be recovered while analyzing range-rate data in arcs of slightly less than 15 minutes (between thrusts). Baselines are refined every 15 minutes using arcs that begin 40 seconds after a thrust and 20 seconds before a thrust (14 minute arcs).

A data point was created every 5 seconds for 30 days. Two versions of the data were created, one with no noise and the other with noise of $1 \mu \mathrm{m} \mathrm{s}^{-1}$. The noiseless version was useful in the verification of the modifications made to the GEODYN software for the new epoch state parameterization. The noiseless version was also used in preliminary studies to determine range-rate data sensitivity to epoch state parameters in orbit refinement solutions. The lack of noise makes residual analysis easier. All gravity recovery simulations used the data with noise.

Most of the studies in this paper involve reducing the simulated data using an a priori gravity field which is different from EGM96. A crucial question is to determine whether the intersatellite rangerate data can be used by themselves (after orbits have been precomputed with a variety of tracking types and then refined in certain components with the intersatellite measurements) to recover the coefficients of EGM96, starting from a different gravity field. The a priori gravity field is a "clone" of EGM96 through degree 70 and is zero above that. At degree 70 and below, the coefficients of the a priori clone field differ from EGM96 by about the standard errors of EGM96. While considerable evidence suggests that the EGM96 errors are realistic (Lemoine et al., 1998a), the EGM96 model itself does differ from some other recent models by amounts exceeding these errors. Our a priori clone gravity model should therefore be considered as fairly "close" to EGM96, so if we are able to recover a field that is closer to EGM96 than the clone, then we will have shown that our technique is useful for recovery of small gravity signal below degree 70 . Basing the test on an a priori field that is already close to EGM96 is a stringent test of sensitivity. Above degree 70, our test will be less severe since we are starting from zero (which is farther away from EGM96 than the standard errors). Even so, we 
will be able to determine if signal above degree 70 can be recovered with our method of reduction.

The clone field was also used to generate the starting trajectory that is used to obtain the a priori elements to begin the orbit refinement process. The intersatellite range-rate data are used in the refinement step to adjust only three or four of the twelve initial state parameters (as discussed below). We accept the other eight or nine elements from the starting trajectory without alteration. So, in some sense, the starting trajectory contains the bulk of the the information about the orbits that will be used to extract gravity signal.

In practice, the starting trajectories will have to be generated with whatever a priori gravity field is available. Our starting trajectory was determined in a manner somewhat approaching "reduced dynamic" methods (e.g., Bertiger et al., 1994; Rowlands et al., 1997). The solution had the following characteristics:

1. The clone gravity field was used.

2. $\Delta V$ 's were solved for at the appropriate times.

3. Empirical, periodic once per revolution accelerations (phase and amplitude parameters) were solved for in both the along- and cross-track components. Phase and amplitude were estimated every 30 minutes.

4. The simulated intersatellite range-rate measurements were used as a tracking data type.

5. To simulate the strong geometrical constraints that GPS tracking would provide, the "truth" ephemeris was used as a tracking data type.

6. Solutions used thirty hours of data, from 0 hours of one day to 6 hours of the next.

The starting ephemeris produced from the above solution differs from the "truth position" in an RMS sense by 3 centimeters (total position). That is probably optimistic for what will actually be attainable 
for the position components of a reduced dynamic trajectory, but the velocity components are probably more important for this study (Jekeli, 2000). The starting trajectory fits the intersatellite range-rate data at $12 \mu \mathrm{ms}^{-1}$, but that is a bit misleading. Although the starting trajectory was produced by reduced dynamic techniques, it will be used to generate a priori initial state elements for the gravity analysis. That analysis cannot make use of the empirical accelerations from the reduced dynamic trajectory (they contain gravity information). The a priori elements for the gravity analysis are gleaned from the starting trajectory and produce fits of over $200 \mu \mathrm{m} \mathrm{s}^{-1}$ (RMS) in a 14 minute arc when they are used without the benefit of the empirical accelerations that were part of the starting trajectory. Our intersatellite range-rate gravity analysis starts with input elements which need to be refined along with the gravity.

Note that empirical accelerations solved for while determining the reduced-dynamic starting trajectory do not alias into the gravity analysis, since the accelerations are used only to determine accurate intial conditions for the second (gravity estimation) step. The jump from 12 to $200 \mu \mathrm{m} \mathrm{s}^{-1}$ mentioned above is a result of removing empirical accelerations in the second step. The $200 \mu \mathrm{m} \mathrm{s}^{-1}$ contains signal from initial state error as well as from gravity errors that had been soaked up by the empirical accelerations.

\section{Baseline Refinement}

Before attempting any large simulations to demonstrate the ability of our technique to recover gravity information, we performed some smaller simulations with the goal of understanding baseline refinement from intersatellite measurements. In particular, we wish to determine which parameters need be refined and which parameters can be taken from the reduced dynamic trajectory without alteration.

Our first set of tests used the truth force model (EGM96 through degree 120) and the noiseless intersatellite range-rate data. These tests were performed on short arcs (14 minutes) between the 
$\Delta V$ thrusting events described in the previous section. The goal of these tests is to find the best minimum set of initial state parameters to estimate so that the a posteriori range rate residuals are well below the $1 \mu \mathrm{ms}^{-1}$ level (if all of the elements are set to truth values, then the noiseless data should fit perfectly when EGM96 is used). Although the data used in these tests were noiseless, they were weighted in the least squares solution as if they had a standard deviation of 1 micron per second. This is noted so that the formal standard deviations of the adjusted parameters can be interpreted.

The first runs adjusted a single parameter. As noted in the previous section, elements taken from the reduced dynamic trajectories produce residuals with an RMS of over $200 \mu \mathrm{ms}^{-1}$. When only a single parameter is adjusted, the only parameter that could reduce the RMS residual to under 100 $\mu \mathrm{m} \mathrm{s}^{-1}$ was the velocity pitch parameter. In fact, the velocity pitch parameter adjustment produced an RMS residual of under $10 \mu \mathrm{ms}^{-1}$.

The second set of runs adjusted two parameters with velocity pitch always being one of the pair. While examining the choice of a second parameter it became clear that over short arcs, two parameters are very correlated: $R_{b v}$ (baseline velocity magnitude) and $R_{m}$ (the distance of the baseline midpoint from the center of mass of the earth). The choice of either of these parameters as the second parameter to accompany velocity pitch produces almost identical residual patterns. When these two parameters $\left(R_{b v}\right.$ and $\left.R_{m}\right)$ are allowed to adjust simultaneously along with velocity pitch, the inverted normal matrix shows a correlation between $R_{m}$ and $R_{b v}$ of very nearly one. When either of these two parameters accompanies velocity pitch, the solution produces an RMS residual of less than $1 \mu \mathrm{ms}^{-1}$. We chose to use velocity magnitude because the adjustments in this component were always less the $100 \mu \mathrm{m} \mathrm{s}^{-1}$ and usually less than $20 \mu \mathrm{m} \mathrm{s}^{-1}$. This seemed more reasonable than the adjustments in the $R_{m}$ parameter (sometimes more than 30 centimeters), since our reduced dynamic trajectories were better than 10 centimeters radially. In general the $R_{m}$ parameter should almost always be determined from a reduced dynamic trajectory to better than 10 centimeters, so over short arcs this parameter is 
not likely to need adjustment.

Even though the adjustment of velocity pitch and velocity magnitude bring the RMS residuals to under a micron per second, there are noticeable trends in the residuals, sometimes near the micron per second level. Because of this we made one more set of runs to search for a third parameter. We find that baseline position pitch works best. With position pitch, velocity magnitude and velocity pitch adjusting, the RMS residuals were well under $0.1 \mu \mathrm{m} \mathrm{s}^{-1}$. Therefore, in our short-arc gravity recovery experiments (next section) we adjusted these three parameters. It may be possible to avoid adjusting position pitch, especially if arcs of 10 minutes or less are attempted.

Our final set of runs deal with longer arcs. In the next section we make two gravity field determinations. One determination uses 30 days of 14 minute arcs (2878 arcs). The other uses 30 arcs, each one day in length. We want to find the proper set of adjusting parameters for one-day arcs. In extending to 2-hour arcs we found that our "14 minute parametrization" (position pitch, velocity magnitude and velocity pitch) held up quite well, producing fits of less than $0.2 \mu \mathrm{m} \mathrm{s}^{-1}$. We also found that for arc lengths of 2 hours, the $R_{m}$ parameter is still highly correlated with baseline velocity magnitude. At an arc length of 12 hours the "14 minute parameterization" produces RMS residuals of close to $1 \mu \mathrm{m} \mathrm{s}^{-1}$. Also, at this arc length, the $R_{m}$ parameter is less correlated (still 0.999) with the velocity magnitude parameter. At 12 hours and above, 4 parameters (including $R_{m}$ ) can be sensibly adjusted. In these adjustments the formal standard deviation of $R_{m}$ is less than a centimeter. When $R_{m}$ is adjusted as the fourth parameter in a 12 hour arc, the RMS residual is reduced to below a $0.1 \mu \mathrm{m} \mathrm{s}^{-1}$. In a 24 hour arc, the correlation between $R_{m}$ and velocity magnitude is reduced to 0.995 . Our "long arc" gravity analysis described in the next section simultaneously adjusts four arc parameters along with gravity coefficients.

As noted above, the noiseless version of the data was used in these baseline refinement studies and the force model was set to "truth" values. So if enough initial state parameters are allowed to 
adjust, the data will be fit perfectly. The gravity analysis described in the next section uses the initial state parameterizations that have just been described, but the clone gravity field is used to compute the trajectories (refine the trajectories before normal equations are generated) and the "noise added" version of the data is used. In this mode, when the three short-arc parameters are allowed to adjust, the arcs fit the the data between 7 and $25 \mu \mathrm{ms}^{-1}$. The day-long arcs, with 4 parameters adjusting, have fits between 20 and $60 \mu \mathrm{ms}^{-1}$.

\section{Gravity Solutions}

The gravity solutions presented in this section are, like most simulations, somewhat optimistic. The simulations include no unmodeled effects other than random measurement noise and some initial satellite state error (which is left over from those initial satellite state elements that we leave unaltered from the reduced dynamic trajectory). In the long-arc analysis we modeled (without adjustment) the "truth" values of the $\Delta V$ thrust events, which implies perfectly performing accelerometers. Futher-

more, in addition to some initial satellite state refinement, only gravity parameters are estimated from the simulated data; no attempt is made to estimate, for example, tidal and gravity parameters simultaneously. In fact, tides and other high-frequency atmospheric and oceanic mass motions pose serious aliasing problems for an SST mission because of the difficulty in modeling and removing the associated gravity effects at required accuracies (Zlotnicki et al., 2000; Verhagen et al., 2000). Such problems are here ignored.

The two solutions presented below, one comprising short arcs and the other comprising long arcs, are intended to be taken qualitatively. The differences between the short-arc solution and the long-arc solution are of particular interest, since they reveal how much information is potentially lost when short arcs are used and what can be gained by extending arc length (assuming the level of unmodeled forces does not preclude the use of long arcs). But the fact that it is possible to obtain sensible gravity solutions from arcs shorter than 15 minutes is significant in itself. 
We estimated two gravity fields using the 30 days of simulated data described in Section 4 . Each gravity field was estimated to degree 120 without any constraints. The method used in estimating the fields differed in the choice of arc length. The "short arc" field used 2878 arcs 14 minutes in length while the "long arc" field consisted of 30 arcs, each a day in length. The short-arc field estimated $2878 \times 3=8634$ arc (orbit) parameters simultaneously with the 14337 gravity coefficients while the long-arc field estimated $30 \times 4=120$ arc parameters. The short-arc field discarded 1 minute of data around each $\Delta V$ (12 points) every 15 minutes, so the short-arc field uses approximately $7 \%$ fewer observations.

The estimated gravity fields should be compared with EGM96, which was used to simulate the data and is therefore the 'true' field, and the EGM96 clone, which was used as the a priori field from which gravity normal equations and initial ephemerides were produced. The figures in this section which pertain to coefficient values show differences from EGM96. If our estimates were perfect, the estimated coefficient differences from EGM96 would be zero. Of course the differences are not zero, but they are much smaller than the differences between EGM96 and the EGM96 clone (see Figure 2 below).

The formal errors of our estimated coefficients cannot be directly compared to EGM96 errors. The standard errors of EGM96 are the result of a complex calibration of weights of the many data types used in its solution (Lerch, 1991). The formal errors of our two estimated fields are simply the diagonals of an inverted normal matrix having a single data type which had been assigned a standard deviation of 1 micron per second. The formal errors of our estimated gravity coefficients should therefore be interpreted only in a relative sense. Internally, they should be reliable for seeing which portions of the estimated gravity fields are more strongly or weakly determined. Externally, they should provide a good basis to compare two gravity fields that were estimated in a largely similar fashion. 
Figure 1 shows the RMS differences of the prior field and the two estimated fields with respect to the 'true' EGM96. It shows immediately (and reassuringly) that both estimated fields are considerable improvements over the a priori field. Neither field appears to be impacted by the truncation of the prior field at degree 70; both show relatively smooth differences with respect to EGM96 through all degrees. More interestingly, Figure 1 emphasizes that an accurate gravity field can be estimated from short arcs. The short-arc gravity field is significantly better than the a priori field at every degree from degree 5 through degree 100 . It is not surprising that a field that is sewn together only from suborbital arcs is weaker at the very lowest degrees. The comparison of the performance (by degree) of the short-arc field with the long-arc field is not unfavorable to the short-arc field from degree 30 upwards. However, at about degree 100, the short-arc field stops outperforming the clone gravity field. This does not happen until about degree 110 for the long-arc field. When judging the relative performance of the two estimated fields, it should be remembered that our simulations assume that the accelerometer is working perfectly (no unmodeled forces), which is much more beneficial to the outcome of the long-arc field than the short-arc field.

More detailed comparisons of the three fields are shown in Figure 2, while the estimated formal errors are shown in Figure 3. Over a wide region of degrees and orders both estimated fields show remarkable improvements relative to the EGM96 clone. Many coefficients are improved by two orders of magnitude. This is generally consistent with figures quoted in the 1997 National Research Council report (see their Figure 2.6), but it is more definitive since the NRC calculations were based on an analytic theory of Jekeli and Rapp (1980) which assumes isotropic data (including data at all inclinations) and ignores possible required arc parameters.

In general, Figures 2 and 3 agree well and show where the solutions are strong and where they are weakened when arcs are shortened. For a given degree, both the short-arc and long-arc fields determine lower order coefficients more accurately than higher order coefficients. That trend is much 
more pronounced in the short-arc field, especially for sectorials, which for some low degrees are actually slightly inferior to the clone field. Again, this is not surprising-one cannot expect an extremely short arc (roughly $1 / 6$ of a revolution) in a high inclination orbit to be sensitive to long-wavelength sectorial terms.

Most remarkably, the long-arc and short-arc fields are very comparable at low orders, especially so for zonal coefficients. The RMS discrepancy over all degrees (2 through 120) between EGM96 and the short-arc field for zonal coefficients is $1.9 \times 10^{-10}$. For the long arc field that discrepancy is $1.8 \times 10^{-10}$. Figure 4 shows that this striking similarity holds for all zonal terms, save the few between degrees 2 and about 8 . For degrees 10 through 40 both long-arc and short-arc zonals are two orders of magnitude (or more) more accurate than the clone model. They are nearly one order of magnitude more accurate at degrees 40 through about 100. The improvement ceases at degree 112. Determining zonal gravity coefficients has historically been problematic in satellite geodesy. Clearly, an SST mission-even one which for one reason or another is restricted to using very short arcs of data-is likely to yield significant advances.

\section{Summary}

We have demonstrated a promising technique for the analysis of low-low intersatellite range-rate data from a gravity mapping mission. The technique largely (but not completely) decouples the task of orbit determination from the task of extracting gravity information. This has several advantages:

1. In the first step of the procedure orbits can be determined using all available tracking types and reduced dynamic techniques. Extraction of gravity information in the second step benefits from the use of empirical accelerations used in the first step without aliasing problems.

2. In the second step of the procedure, only intersatellite data are used. Gravity information can be extracted without complex solutions involving multiple data types. 
3. Because orbits are only refined (not fully determined) in the second step, short arc analysis is facilitated.

The technique transforms the standard 12 initial epoch state vector parameters of the two satellites into spherical coordinates describing the baseline between the two satellites. We have performed an analysis to show which of these 12 parameters need to be estimated simultaneously with gravity coefficients.

We have used this technique to estimate gravity fields from simulated data. Our study neglects many effects, but unlike many studies of gravity missions in the literature, we consider the need to estimate orbit parameters simultaneously with gravity coefficients. We have investigated the possibility of estimating a gravity field entirely from short arcs (14 minutes) of intersatellite range rate data and found that this indeed should be possible. We have also shown the differences between a short-arc gravity field and a field estimated from long arcs. The use of long arcs (if possible) adds information primarily at the higher orders of every degree of the gravity field.

Acknowledgments. We thank our colleagues Steve Klosko and Scott Luthcke for general discussions on satellite-satellite tracking and for specific discussions on this paper. Computational facilities were provided by the NASA Center for Computational Sciences at Goddard Space Flight Center. 


\section{References}

Bertiger W I, et al. (1994) GPS precise tracking of Topex/Poseidon: results and implications, J. Geophys. Res., 99: 24449-24464.

Cui C, Lelgemann D (2000) On non-linear low-low SST observation equations for the determination of the geopotential based on an analytical solution, $J$. Geod., 74: 431-440.

Colombo, O L (1984) The global mapping of gravity with two satellites, Netherlands Geodetic Commission, Delft, $253 \mathrm{pp}$.

Douglas B C, Goad C C, Morrison F F (1980) Determination of the geopotential from satellite-to-satellite tracking data, J. Geophys. Res., 85: 5471-5480.

Jekeli, C (1999) The determination of gravitational potential differences from satellite-to-satellite tracking, Celes. Mech. Dyn. Astro., 75: 85-101.

Jekeli C, Rapp R H (1980) Accuracy of the determination of mean anomalies and mean geoid undulations from a satellite gravity field mapping mission, Ohio Stat Univ. Geod. Sci. Rep. 307, Columbus, 22 pp.

Jekeli C, Upadhyay T N (1990) Gravity estimation from STAGE, a satellite-to-satellite tracking mission, $J$. Geophys. Res., 95: 10973-10985.

Kahn W D, Klosko S M, Wells W T (1982) Mean gravity anomalies from a combination of Apollo/ATS-6 and GEOS-3/ATS-6 SST tracking campaigns, J. Geophys. Res., 87: 2904-2918.

Kaula W M (1983) Inference of variations in the gravity field from satellite-to-satellite range rate, J. Geophys. Res., 88: 8345-8349.

Lemoine F G, et al. (1998a) The development of the joint NASA GSFC and NIMA geopotential model EGM96, NASA Tech. Memo. 206861, Goddard Space Flight Center, Greenbelt, 575 pp.

Lemoine F G, Cox C M, Chinn D S, Pavlis N K, Torrence M H, Pavlis E C, Williamson R G (1998b) Improved Earth gravity solutions from TDRSS tracking (abstract), Ann. Geophysicae, xxxxxxxxxxxx.

Lerch, F J (1991) Optimum data weighting and error calibration for estimation of gravitational parameters, Bull. Géod., 65: 44-52. 
National Research Council (1997) Satellite Gravity and the Geosphere, National Academy Press, Washington, $112 \mathrm{pp}$.

Pavlis D E, Poulose S, Rowton S C, McCarthy J J (2001) GEODYN II System Documentation, Raytheon ITSS Contr. Rep., Greenbelt, MD.

Rowlands D D, Luthcke S B, Marshall J A, Cox C M, Williamson R G, Rowton S C (1997) Space shuttle precision orbit determination in support of SLA-1 using TDRSS and GPS tracking data, J. Astronaut. Sci., 113-129.

Schwintzer P, Reigber C, Barthelmes F, Bode A, Gruber T, Koenig R, Biancale R, Balmino G, Lemoine J-M, Loyer S, Perosanz F (2000) Gravity field recovery from CHAMP: first results (abstract), Eos, Trans. Am. Geophys, Union, 81: F307.

Tapley B D, Reigber C (2000) The GRACE mission: status and future plans (abstract), Eos, Trans. Am. Geophys. Union, 81: F307.

Verhagen A A, Schrama E J O, Bouman J (2000) Time variations in the gravity field due to atmospheric pressure variations (abstract), Eos, Trans. Am. Geophys. Union, 81: F311.

Wagner C A (1983) Direct determination of gravitational harmonics from low-low Gravsat data, J. Geophys. Res., 88: 309-321.

Wagner C A (1987) Improved gravitational recovery from a geopotential research mission satellite pair flying en echelon, J. Geophys. Res., 92: 8147-8155.

Wolf M (1969) Direct measurements of the earth's gravity potential using a satellite pair, J. Geophys. Res., 74: 5292-5300.

Zlotnicki V, Fukumori I, Hirose N, Menemenlis D, Ali A (2000) Dealiasing GRACE: the high-frequency ocean response to wind and pressure (abstract), Eos, Trans. Am. Geophys. Union, 81: F308. 
Table 1: Initial Keplerian Elements for Both Satellites

\begin{tabular}{lc}
\hline Semimajor axis $a$ & $6878050 \mathrm{~m}$ \\
Eccentricity $e$ & 0.001 \\
Inclination $i$ & $89.1^{\circ}$ \\
Node $\Omega$ & $0^{\circ}$ \\
Perigee argument $\varpi$ & $0^{\circ}$ \\
Mean anomaly $m$ & $0^{\circ}$ \\
Period $T$ & $94.6 \mathrm{~min}$ \\
\hline
\end{tabular}

Note: Initial epoch satellite $2=$ initial epoch satellite $1+30$ seconds. 


\section{FIGURE CAPTIONS}

Figure 1. RMS gravity coefficient differences with respect to a "true" gravity field of (solid line) the a priori field, (dashed line) a solution employing short arcs, and (dotted line) a solution employing long arcs. The discontinuity in the a priori model arises because it is truncated to zero above degree 70.

Figure 2. Results of gravity inversion simulations showing $\log \sqrt{ }\left(\Delta C_{n m}^{2}+\Delta S_{n m}^{2}\right)$, for fully normalized Stokes coefficients $C_{n m}, S_{n m}$ differenced with the "true" gravity field, for (a) the a priori gravity field, (b) the short-arc gravity inversion, and (c) the long-arc gravity inversion. The discontinuity in the a priori model arises because it is truncated to zero above degree 70 . Below degree 70 (a) is indicative of present-day uncertainties in the geopotential, as represented by the standard errors of EGM96.

Figure 3. Formal errors for gravity simulations employing (a) short arcs and (b) long arcs. Colors show the logarithm of errors for fully normalized coefficients.

Figure 4. Differences with respect to a "true" gravity field of zonal coefficients $J_{n}$ of degree $n$, for (solid line) the a priori field, (dashed line) the short-arc gravity solution, and (dotted line) the long-arc gravity solution. All three lines have been smoothed to remove minor statistical variability. The long-arc and short-arc solutions are similar except for the lowest degrees where the short-arc solution is less accurate. 


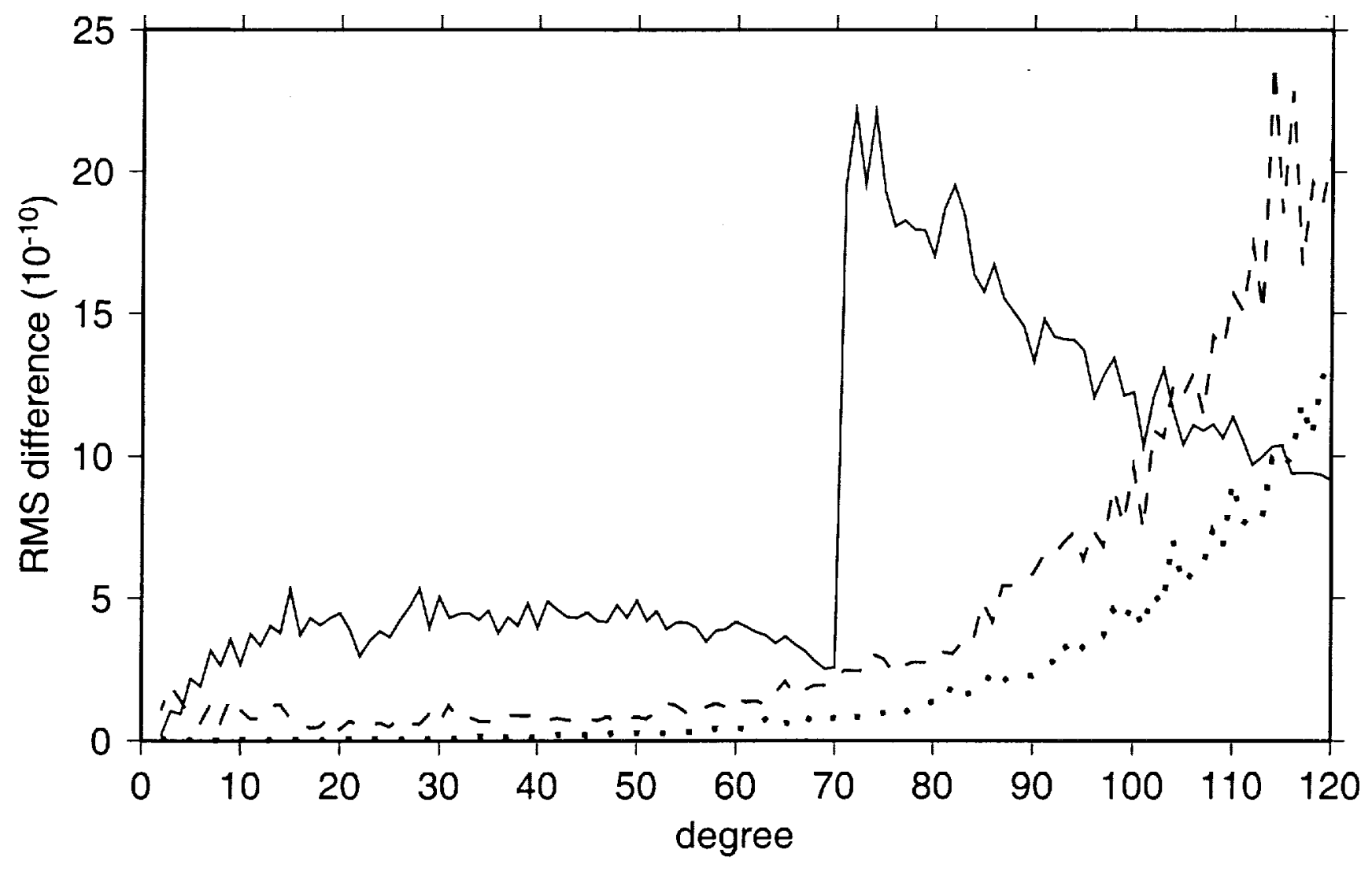

Fig. 1 

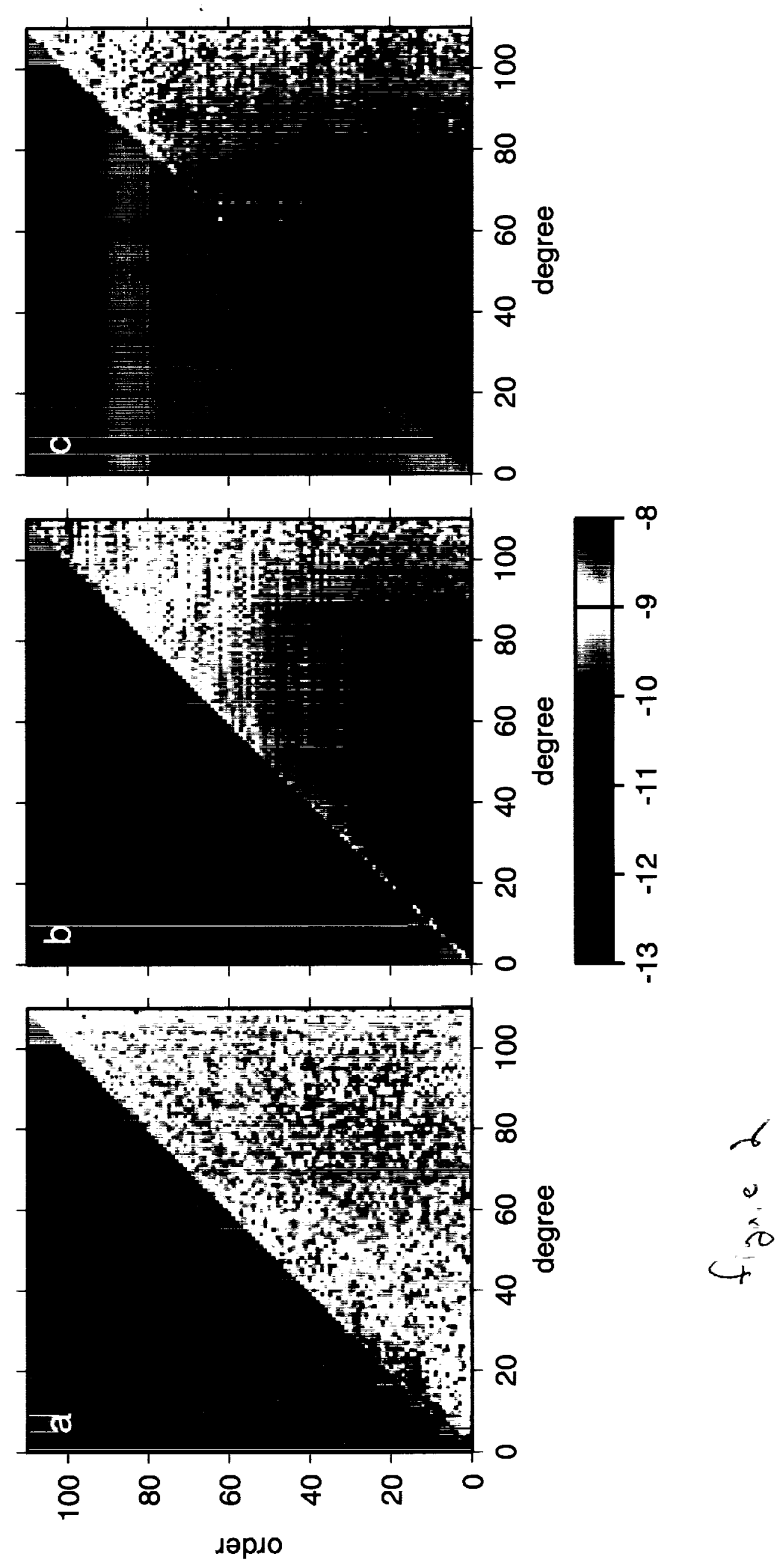


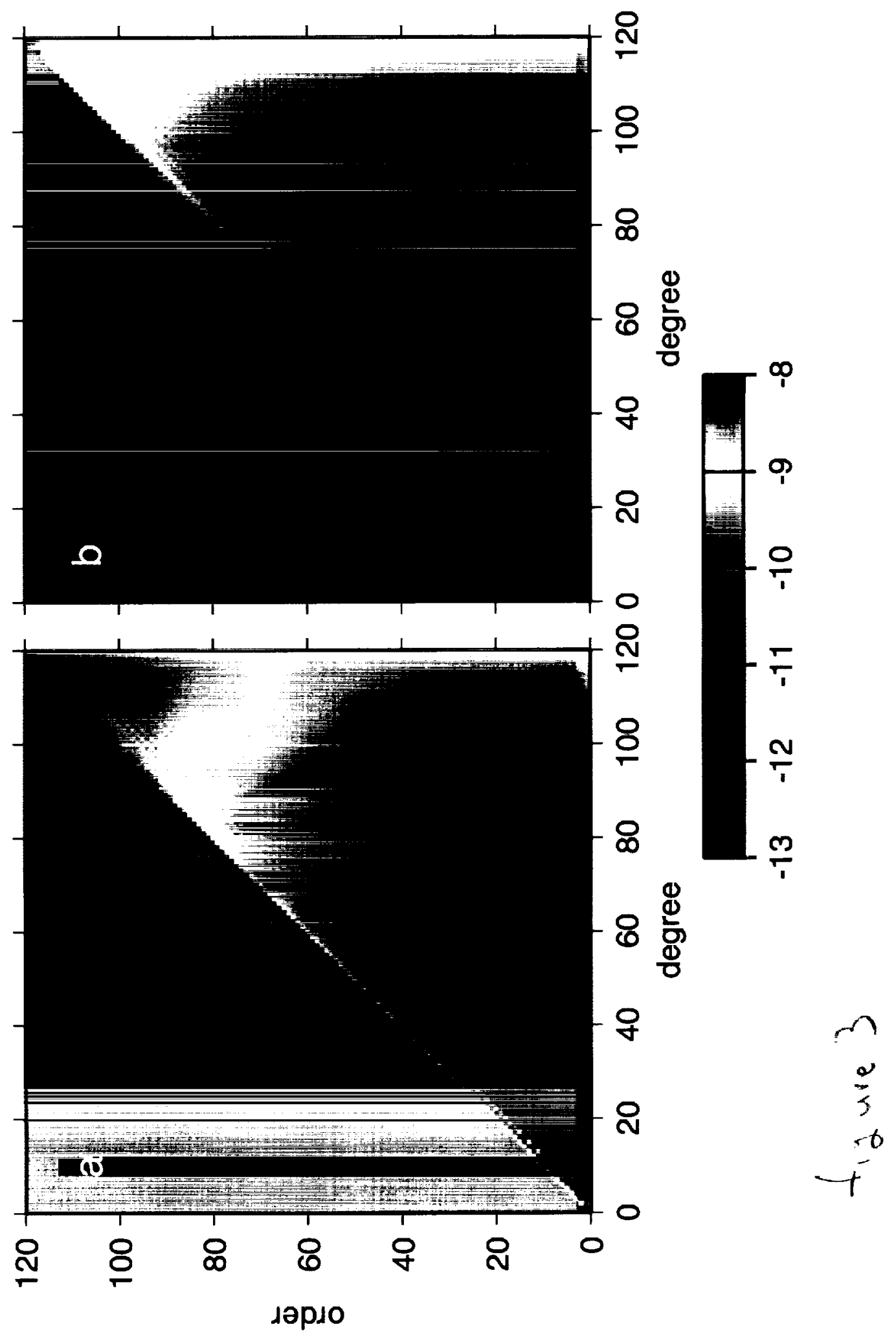




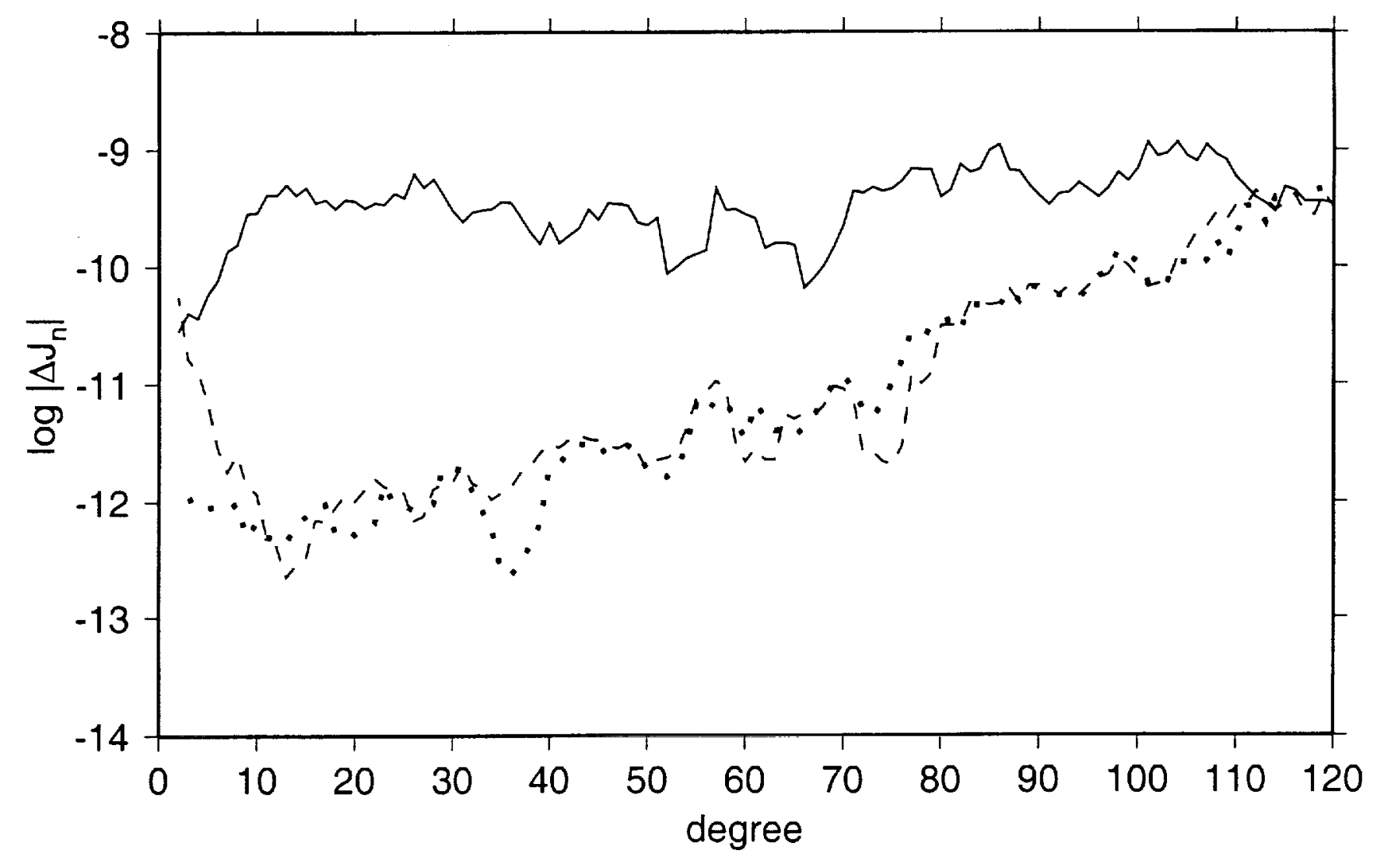

Fig. 4 